Article

\title{
Older adults rate their mental health better than their general health
}

\author{
Elena M. Magwene, ${ }^{1}$ Ana R. Quiñones, ${ }^{2}$ Gillian L. Marshall, ${ }^{3}$ Lena K. Makaroun, ${ }^{3,4}$ \\ Stephen Thielke 3,5 \\ ${ }^{1}$ Mental Health Service, VA Puget Sound Health Care System, Seattle, WA; ${ }^{2}$ Oregon Health and \\ Science University, Portland, OR; ${ }^{3}$ University of Washington, Seattle, WA; ${ }^{4}$ VA Health Services \\ Research and Development, VA Puget Sound Healthcare System, Seattle, WA; ${ }^{5}$ Geriatric Research, \\ Education, and Clinical Center, VA Puget Sound Health Care System, Seattle, WA, USA
}

\begin{abstract}
Significance for public health
Self-rated health (SRH) has become established as a general measure of health status, but less is known about self-rated mental health (SRMH). Recent epidemiological studies have included self-rated mental health (SRMH) without scrutinizing its properties and in particular its relationship with SRH. In a large dataset of Medicare recipients, we found that self-rated mental health was consistently rated better than self-rated health, across all patient groups. None of the sociodemographic or health factors we examined accounted for this discrepancy. Self-rated mental health seemed to be more resistant to the effects of medical illnesses and functional impairments than was self-rated health. This points to a likely difference in how people formulate and differentiate between their mental and general health, with mental health being seen as more separate from other health factors. These findings encourage caution in the use of SRMH in analytic models, especially if included simultaneously with SRH.
\end{abstract}

\section{Abstract}

Background. Self-rated health (SRH) shows strong associations with measures of health and well-being. Increasingly, studies have used self-rated mental health (SRMH) as a predictor of various outcomes, independently or together with SRH. Research has not firmly established if and how these two constructs differ. We sought to characterize the relationship between SRH and SRMH, and to determine how this relationship differed across subgroups defined by sociodemographic and health-related characteristics.

Design and methods. We analyzed data from the 2012 CAHPS Medicare Advantage Survey. SRH and SRMH ratings were crosstabulated to determine the distribution of responses across response categories. The expected joint probability distribution was computed and compared to the observed distribution. A constructed variable indicated whether SRMH was better, the same, or worse than SRH. We analyzed the distribution of this variable across various subgroups defined by sociodemographic and health-related factors.

Results. A total of 114,905 Medicare Advantage beneficiaries responded to both the SRH and SRMH questions. Both in general and within all subgroups, SRMH was usually rated as better than $\mathrm{SRH}$, and rarely as worse.

Conclusions. Within a large group of Medicare recipients, the overwhelming trend was for recipients to rate their mental health as at least as good as their overall health, regardless of any sociodemographic and health-related factors. This finding of a shifted distribution encourages caution in the analytic use of selfrated mental health, particularly the use of both SRH and SRMH for adjustment. Additional research is needed to help clarify the complex relationship between these variables.

\section{Introduction}

Self-rated health (SRH) has been shown to be an important predictor of health and well-being, having associations with a variety of health outcomes including mortality, health care utilization, and functioning. 1,2 The standard item used to measure SRH asks How would you rate your health in general? with response options of excellent, very good, good, fair, and poor. This framework has been extended to a similar question regarding self-rated mental health (SRMH), In general, how would you rate your overall mental health? Less is known about the properties and associations of SRMH, in particular its relationship with SRH.

There has been an increasing use of SRMH in epidemiological and health services studies. It has been applied as a measure of individual health need and of symptom burden. ${ }^{3,4}$ Studies have examined the relationship between SRMH and other mental health measures or conditions, ${ }^{5,6}$ physical health problems, ${ }^{7,8}$ social determinants of health, ${ }^{9}$ and use of health services. ${ }^{3,10}$ This work has not examined in detail how SRH and SRMH are related.

To date, there has been one review published on $\mathrm{SRMH}^{11}$ which examined 57 studies that utilized SRMH, usually as a covariate rather than the object of investigation. This review found that SRMH was moderately correlated with various mental health scales that assessed psychological distress, depression, and general psychiatric symptoms. It also indicated that poor SRMH was associated with increased service utilization, less satisfaction with mental health services, and more physical health problems. Some of the studies reviewed controlled for SRH, and others did not.

One of the studies included in this review discussed the association between SRH and SRMH. Fleishman and Zuvekas found that both SRH and SRMH have independent associations with measures of physical and emotional role functioning, ${ }^{12}$ but SRH has a stronger association with physical role functioning and SRMH has a stronger association with emotional role functioning. The researchers concluded that SRH and SRMH were more strongly correlated with each other than with any of the physical or emotional role functioning measures $(\mathrm{r}=0.54)$. Only one other study (which was not included in the review) has addressed the contribution of SRMH to self-rated overall health. In work by Levinson and Kaplan, ${ }^{13}$ both self-rated physical and mental health were more important in predicting self-rated overall health than other health status indicators, including comorbidities, mental disorders, and disability. Nevertheless, SRMH was twice as impor- 
tant in predicting self-rated overall health as was self-rated physical health. The authors concluded that the mental component in the self-rating of overall health is stronger than the physical one. This work suggests differences in the underpinnings for two self-rated health measures, but does not clarify the relationship between SRH and SRMH. Prior research has therefore not established systematically whether SRH and SRMH represent different constructs, and exactly how they differ. Missing from the analyses are straightforward descriptive results that compare the responses to both questions. How often do people say their SRMH is the same as, better than, or worse than their SRH? What sorts of individuals describe SRMH as being better than SRH, and vice versa? Correlation and regression coefficients usually fail to account for this relationship. Because it can be difficult to represent and discuss the relationship between two self-report variables, we propose a simplifying analogy. SRH asks about general health, which is assumed to include various subcomponents, including a mental one. This is a part-towhole relationship, using two subjective ratings. A similar relationship exists between a diner's ratings for (1) the dinner as a whole, and (2) dessert (or some other subcomponent of dinner). Fully characterizing the ratings for dessert requires comparing them to the ratings for the entire meal and vice versa. Looking at both variables can answer general questions about the influence of one rating on the other. For instance, how frequently people perceive dessert as better or worse than the meal as a whole, how often the ratings are identical, or if a poor dessert ruins dinner. Such an analogy illustrates the modeling challenge, and helps to clarify a limitation inherent in the part-to-whole relationship, namely that the two self-rated health questions do not differentiate between parts: physical (but not mental) and mental (but not physical) health. To characterize the relationship between the two self-rated health items, we sought to ascertain the relative responses (better, same, or worse) between SRMH and SRH questions among a large group of Medicare recipients. We hypothesized, based on previous research, that there would be a strong association between SRMH and SRH, and that any differences between them would not be skewed (i.e. that one would not be consistently rated better or worse than the other). In addition, we hypothesized that subgroups defined by sociodemographic and health characteristics would show different patterns in the comparative ratings of SRMH and SRH. Prior studies did not give strong support for hypothesizing a specific direction to the relationship in subgroups.

\section{Materials and Methods}

Data came from one wave of the Consumer Assessments of Healthcare Providers and Systems (CAHPS $\AA$ ) Survey, administered in 2012 (Cohort 13). Participants were enrolled in one of several the Medicare Advantage (MA) programs, which provide private health insurance to about $30 \%$ of Medicare beneficiaries. Every year, each of the MA contracts surveys 800 randomly-selected respondents. The 12-page core survey was sent by mail, with phone follow-up for non-responders. Spanish-language surveys were sent to those who returned a postcard indicating this preference. The average age of the sample was 72.8 years, and $55.5 \%$ were female. $13 \%$ indicated non-white race, and $52.5 \%$ reported some college education. Additional, detailed information about survey methods, composition of the cohort, and nonresponse patterns have been published elsewhere. ${ }^{14-18} 13.7 \%$ of the MA recipients were younger than age 65 , based on disability status. We included them in the analyses because we could not assume that either disability status or age would influence the relationships in question. We assumed that differences in this group would appear in stratified analyses.

\section{Measures}

\section{Self-rated health}

The SRH item asked, In general, how would you rate your overall health? with responses of Excellent, Very Good, Good, Fair, or Poor.

\section{Self-rated mental health}

The SRMH item (which appeared immediately after the SRH item in the survey) asked, In general, how would you rate your overall mental or emotional health [emphasis retained], with the same response categories.

\section{Sociodemographic characteristics}

The CAHPS administrative dataset identifies respondent gender and age. The survey asked about highest education level $\left(8^{\text {th }}\right.$ grade or less; some high school, but did not graduate; high school graduate or GED; some college or 2-year degree; 4-year college graduate; and more than 4-year college degree), ethnicity (Hispanic or Latino origin or descent), and race (with a request to select one or more from White; Black or African-American; Asian; Native Hawaiian or other Pacific Islander; and American Indian or Alaska Native).

\section{Health variables}

Four other health-related variables which might be associated with differential response in SRH and SRMH were used to tabulate descriptive results.

Comorbidities were assessed asking the question, Has a doctor ever told you that you had any of the following conditions?, with responses for heart attack, angina or coronary artery disease, stroke, cancer other than skin cancer, lung disease, or any kind of diabetes or high blood sugar. These were summed to produce a comorbidity score ranging from 0-6.

Activities of daily living were assessed asking the question, Because of a health or physical problem are you unable to do or have any difficulty doing the following activities. The domains were bathing, dressing, eating, getting in or out of chairs, walking, and using the toilet. The response options were I am unable to do this activity, Yes, I have difficulty, and No, I do not have difficulty. Respondents who were unable to perform the activity or performed the activity with difficulty were coded as having an impairment. Individual impairments were summed to produce an ADL impairment score ranging from 0-6.

Health care utilization was assessed asking the question, In the last 6 months, not counting the times you went to an emergency room, how many times did you go to a doctor's office or clinic to get health care for yourself? Responses were none, 1, 2, 3, 4, 5 to 9 , and 10 or more. The use of urgent services was coded as a binary variable and asked whether the respondent had an illness, injury, or condition that needed care right away in a clinic, emergency room, or doctor's office in the last six months.

\section{Analysis}

We excluded cases which did not include a response to both the SRMH and SRH items. If other parts of the survey were missing, each case was excluded only from the analyses that used those items. We cross-tabulated SRH and SRMH ratings to determine the distribution of responses across response categories. We calculated the Spearman's rank-order correlation. We computed the 
expected joint probability distribution, if SRH and SRMH were independent variables. The predicted joint probability distribution was calculated using a five-by-five table, with the predicted values determined by what one would anticipate if SRH and SRMH were independent. The row percentages (i.e. SRH, for each of the five response categories) were multiplied by the column percentages (i.e. SRMH, for each of the five response categories). These were compared with the observed percentages. We produced tables characterizing the sample on the basis of the sociodemographic, outcome, and predictor variables. We constructed a variable indicating whether SRMH was better, the same, or worse than SRH, and constructed distributions to show the relative frequencies of this by SRH and SRMH category. We then tabulated the frequency of better, same, or worse SRMH using the response categories in the sociodemographic and predictor variables.

Analyses were conducted in 2016 using SPSS (IBM Corp. Released 2010. IBM SPSS Statistics for Windows, Version 19.0. Armonk, NY: IBM Corp.). The University of Washington Institutional Review Board determined that this project was not human subjects research.

\section{Results}

Surveys were mailed to 274,996 MA beneficiaries. A total of 118,444 surveys were returned. Of these, 114,905 included responses to both the SRH and SRMH questions.

Figure 1 shows the unadjusted distribution of responses to the SRMH and SRH categories. SRMH showed higher scores overall.

Table 1 is a cross-tabulation of the number of respondents within each SRH and SRMH category. The majority of respondents $(64.6 \%)$ reported excellent or very good SRMH, while only $34.3 \%$ of respondents fell into the same SRH categories. The majority of respondents (62.5\%) reported SRH to be very good or good. Additionally, the vast majority $(78.7 \%)$ of those with fair/poor SRMH also had fair/poor SRH. In contrast, only $30.1 \%$ of those with fair/poor SRH also had fair/poor SRMH. Thus, most respondents with fair/poor SRMH also rated their SRH as fair/poor, but this reverse did not hold true.

Figure 2 shows a side-by-side comparison. On the left is the predicted joint probability distribution, if SRH and SRMH were independent. On the right is the observed distribution. More than half of participants reported SRMH to be better than SRH, and few reported it to be worse. A far smaller proportion of respondents than predicted reported worse SRMH as compared to SRH $(6.1 \%$ vs. $19.6 \%)$, more than predicted reported the same SRMH as SRH (38.1\% vs. $22.3 \%$ ), and a similar number reported better SRMH and SRH (55.8\% vs. 58.1\%). The Spearman's rank-order correlation for SRMH and SRH was 0.5063 .
Table 2 shows the comparative ratings based on subcategories. The second column indicates the prevalence of the variable. The third and fourth columns show the mean SRMH and SRH ratings within that group. Across all variables, the SRMH rating was better (lower number) than the SRH rating by approximately $0.5-1$ point on the $1-5$ scale.

The fifth through seventh columns of the table show the proportion in each group reporting better, the same, or worse SRMH compared to SRH. With only a few exceptions, more than half the participants reported their SRMH to be better than their SRH across all subgroups. With one exception (age $<55$ years), no more than $10 \%$ of the respondents reported their SRMH to be worse than their SRH. For the entire group, 55.5\% reported better SRMH than $\mathrm{SRH}, 6.1 \%$ reported worse SRMH, and $38.1 \%$ reported it to be the same. Among the health-related variables, with greater numbers of

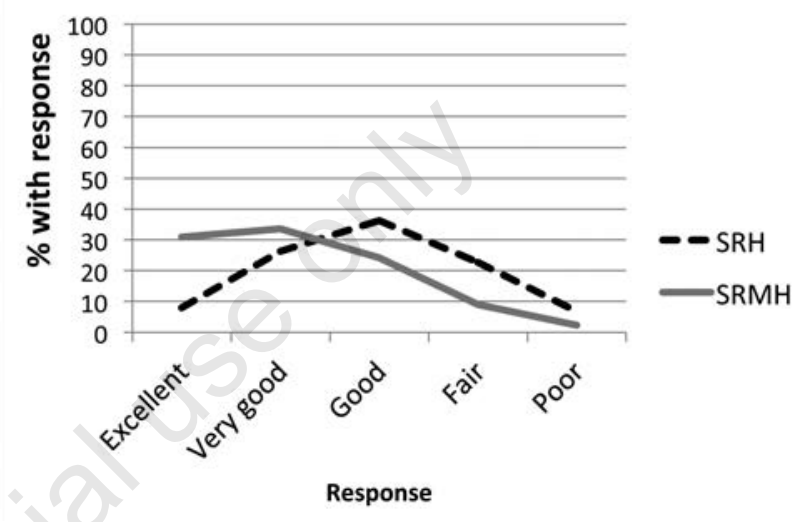

Figure 1. Unadjusted distribution of responses to self-rated mental health (SRMH) and self-rated health (SRH) categories.

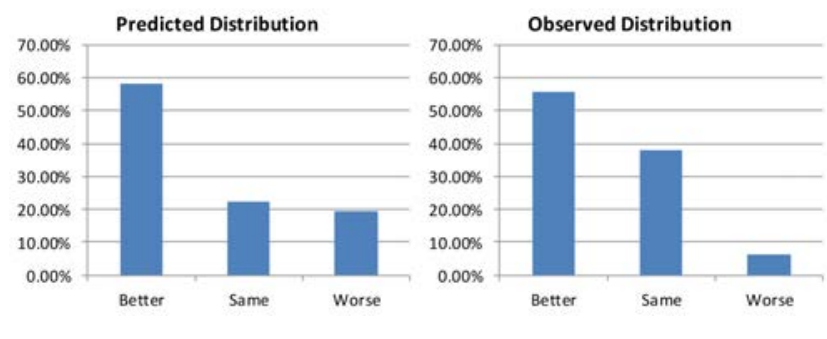

Figure 2. Predicted and observed distributions of self-rated mental health (SRMH) and self-rated health (SRH). Better means that SRMH was better than SRH.

Table 1. Self-rated health and self-rated mental health rating cross-tabulation.

\begin{tabular}{lcccccc} 
& Excellent & Very good & Good & Fair & Poor & Total \\
Excellent & 7637 & 1017 & 372 & 104 & 29 & 9159 \\
Very good & 13,078 & 14,718 & 1958 & 409 & 32 & 235 \\
\hline Good & 10,119 & 14,982 & 14,452 & 1850 & 283 & 41,686 \\
Fair & 3924 & 6699 & 8837 & 5660 & 928 & 26,048 \\
Poor & 734 & 1273 & 2175 & 2274 & 1320 \\
Total & 35,492 & 38,689 & 27,794 & 10,298 & 2632 & 1777 \\
\hline
\end{tabular}

Column totals represent those for SRMH. Row totals represent those for SRH 
Table 2. Ratings of self-rated mental health (SRMH) and self-rated health (SRH), and their comparison, by subcategory.

\begin{tabular}{|c|c|c|c|c|c|c|}
\hline Variable & $\begin{array}{c}\text { Prevalence, } \\
\%\end{array}$ & $\begin{array}{c}\text { Mean* SRMH } \\
\text { (SD) }\end{array}$ & $\begin{array}{c}\text { Mean* SRH } \\
\text { (SD) }\end{array}$ & $\begin{array}{c}\text { SRMH Better } \\
(\%)\end{array}$ & $\begin{array}{c}\text { SRMH Same } \\
(\%)\end{array}$ & $\begin{array}{c}\text { SRMH Worse } \\
(\%)\end{array}$ \\
\hline Entire Group & - & $2.2(1.0)$ & $2.9(1.0)$ & 55.8 & 38.1 & 6.1 \\
\hline \multicolumn{7}{|c|}{ Socio-demographic characteristics } \\
\hline $\begin{array}{l}\text { Age } \\
\qquad \begin{array}{l}<55 \\
55-64 \\
65-69 \\
70-74 \\
75-79 \\
80-84 \\
85+\end{array}\end{array}$ & $\begin{array}{c}4.9 \\
6.8 \\
26.6 \\
21.5 \\
16.5 \\
12.8 \\
10.9\end{array}$ & $\begin{array}{l}3.1(1.2) \\
2.9(1.2) \\
1.9(0.9) \\
2.0(0.9) \\
2.1(1.0) \\
2.2(1.0) \\
2.4(1.1)\end{array}$ & $\begin{array}{l}3.7(1.0) \\
3.8(0.9) \\
2.7(1.0) \\
2.7(1.0) \\
2.9(1.0) \\
3.0(1.0) \\
3.1(1.0)\end{array}$ & $\begin{array}{l}49.4 \\
58.7 \\
54.8 \\
56.5 \\
57.7 \\
56.4 \\
53.7\end{array}$ & $\begin{array}{l}35.5 \\
32.1 \\
40.4 \\
39.0 \\
37.3 \\
37.4 \\
37.7\end{array}$ & $\begin{array}{l}15.1 \\
9.1 \\
4.8 \\
4.5 \\
5.0 \\
6.2 \\
8.6\end{array}$ \\
\hline $\begin{array}{l}\text { Gender } \\
\text { Female } \\
\text { Male }\end{array}$ & $\begin{array}{l}55.5 \\
44.5\end{array}$ & $\begin{array}{l}2.2(1.0) \\
2.2(1.0)\end{array}$ & $\begin{array}{l}2.9(1.0) \\
2.9(1.0)\end{array}$ & $\begin{array}{l}56.3 \\
55.2\end{array}$ & $\begin{array}{l}37.8 \\
38.4\end{array}$ & $\begin{array}{l}5.9 \\
6.4\end{array}$ \\
\hline $\begin{array}{l}\text { Highest education level } \\
8^{\text {th }} \text { grade or less } \\
\text { Some high school } \\
\text { High school graduate/GED } \\
\text { Some college/2 yr degree } \\
\text { 4-year college graduate } \\
\text { More than 4-year college degree }\end{array}$ & $\begin{array}{r}6.0 \\
9.0 \\
32.5 \\
26.5 \\
11.0 \\
15.0\end{array}$ & $\begin{array}{l}2.8(1.1) \\
2.5(1.1) \\
2.3(1.0) \\
2.1(1.0) \\
2.0(1.0) \\
1.8(0.9)\end{array}$ & $\begin{array}{l}3.4(1.1) \\
3.3(1.0) \\
3.1(1.0) \\
2.9(1.0) \\
2.7(1.0) \\
2.5(1.0)\end{array}$ & $\begin{array}{l}51.2 \\
56.5 \\
56.4 \\
57.2 \\
54.2 \\
54.2\end{array}$ & $\begin{array}{l}39.6 \\
36.5 \\
37.6 \\
37.0 \\
39.8 \\
40.4\end{array}$ & $\begin{array}{l}9.2 \\
7.0 \\
6.0 \\
5.8 \\
6.1 \\
5.4\end{array}$ \\
\hline $\begin{array}{l}\text { Ethnicity } \\
\text { Hispanic/Latino } \\
\text { Non-Hispanic/Latino }\end{array}$ & $\begin{array}{c}5.6 \\
94.4\end{array}$ & $\begin{array}{l}2.5(1.2) \\
2.2(1.0)\end{array}$ & $\begin{array}{l}3.2(1.1) \\
2.9(1.0)\end{array}$ & $\begin{array}{l}52.3 \\
56.1\end{array}$ & $\begin{array}{l}38.7 \\
38.0\end{array}$ & $\begin{array}{l}8.9 \\
6.0\end{array}$ \\
\hline $\begin{array}{l}\text { Race } \\
\text { White } \\
\text { Black/African-American } \\
\text { Asian } \\
\text { Native Hawaiian/Pacific Islander } \\
\text { American Indian/Alaska Native }\end{array}$ & $\begin{array}{c}89.3 \\
8.1 \\
2.4 \\
0.4 \\
2.1\end{array}$ & $\begin{array}{l}2.2(1.0) \\
2.4(1.1) \\
2.3(1.0) \\
2.3(1.1) \\
2.5(1.1)\end{array}$ & $\begin{array}{l}2.9(1.0) \\
3.2(1.0) \\
2.9(1.0) \\
3.1(1.1) \\
3.3(1.1)\end{array}$ & $\begin{array}{l}55.9 \\
57.5 \\
50.7 \\
57.0 \\
55.4\end{array}$ & $\begin{array}{l}38.2 \\
35.0 \\
43.5 \\
35.2 \\
36.4\end{array}$ & $\begin{array}{l}6.0 \\
7.4 \\
5.8 \\
7.7 \\
8.2\end{array}$ \\
\hline & & Hea & variables & & & \\
\hline $\begin{array}{l}\text { No. comorbidities } \\
0 \\
1 \\
2 \\
3 \\
4 \\
5 \\
6\end{array}$ & $\begin{array}{l}48.3 \\
30.3 \\
13.5 \\
5.5 \\
1.9 \\
0.4 \\
0.1\end{array}$ & $\begin{array}{l}2.0(1.0) \\
2.2(1.0) \\
2.3(1.1) \\
2.5(1.1) \\
2.6(1.1) \\
2.7(1.1) \\
2.0(1.0)\end{array}$ & $\begin{array}{l}2.5(1.0) \\
3.0(1.0) \\
3.3(1.0) \\
3.6(0.9) \\
3.9(0.9) \\
4.1(0.9) \\
2.5(1.0)\end{array}$ & $\begin{array}{l}45.0 \\
58.7 \\
66.4 \\
71.6 \\
72.5 \\
78.7 \\
63.3\end{array}$ & $\begin{array}{l}47.4 \\
35.5 \\
28.7 \\
24.4 \\
23.3 \\
18.0 \\
30.4\end{array}$ & $\begin{array}{l}7.6 \\
5.8 \\
5.0 \\
4.0 \\
4.2 \\
3.4 \\
6.3\end{array}$ \\
\hline $\begin{array}{l}\text { No. of ADL impairments } \\
0 \\
1 \\
2 \\
3 \\
4 \\
5 \\
6\end{array}$ & $\begin{array}{l}68.5 \\
10.6 \\
7.8 \\
3.6 \\
2.8 \\
2.6 \\
4.0\end{array}$ & $\begin{array}{l}1.9(0.9) \\
2.4(1.0) \\
2.6(1.1) \\
2.8(1.1) \\
3.0(1.1) \\
3.0(1.1) \\
1.9(0.9)\end{array}$ & $\begin{array}{l}2.6(0.9) \\
3.3(0.9) \\
3.6(0.9) \\
3.8(0.9) \\
4.0(0.9) \\
4.1(0.9) \\
2.6(0.9)\end{array}$ & $\begin{array}{l}52.0 \\
64.6 \\
65.9 \\
64.0 \\
64.2 \\
63.7 \\
54.7 \\
\end{array}$ & $\begin{array}{l}42.2 \\
29.2 \\
27.7 \\
28.8 \\
28.7 \\
28.8 \\
37.2\end{array}$ & $\begin{array}{l}5.7 \\
6.2 \\
6.5 \\
7.2 \\
7.1 \\
7.5 \\
8.1\end{array}$ \\
\hline $\begin{array}{l}\text { No. of visits to personal MD } \\
0 \\
1 \\
2 \\
3 \\
4 \\
5 \text { to } 9 \\
10 \text { or more }\end{array}$ & $\begin{array}{l}12.2 \\
30.7 \\
26.1 \\
11.8 \\
6.1 \\
6.8 \\
1.3\end{array}$ & $\begin{array}{l}2.0(1.0) \\
2.0(1.0) \\
2.2(1.0) \\
2.3(1.1) \\
2.4(1.1) \\
2.6(1.1) \\
2.7(1.2)\end{array}$ & $\begin{array}{l}2.5(1.0) \\
2.7(1.0) \\
3.0(1.0) \\
3.2(1.0) \\
3.3(1.0) \\
3.6(1.0) \\
3.8(1.1)\end{array}$ & $\begin{array}{l}45.2 \\
52.9 \\
59.1 \\
61.8 \\
62.6 \\
64.9 \\
64.7\end{array}$ & $\begin{array}{l}47.9 \\
41.5 \\
35.3 \\
32.0 \\
31.5 \\
28.5 \\
30.0\end{array}$ & $\begin{array}{l}6.9 \\
5.6 \\
5.6 \\
6.2 \\
6.0 \\
6.6 \\
5.3\end{array}$ \\
\hline $\begin{array}{l}\text { Needed care right away } \\
\text { Yes } \\
\text { No }\end{array}$ & $\begin{array}{l}34.4 \\
65.6\end{array}$ & $\begin{array}{l}2.3(1.1) \\
2.1(1.0)\end{array}$ & $\begin{array}{l}3.3(1.0) \\
2.8(1.0)\end{array}$ & $\begin{array}{l}62.3 \\
52.4\end{array}$ & $\begin{array}{l}31.8 \\
41.4\end{array}$ & $\begin{array}{l}5.9 \\
6.2\end{array}$ \\
\hline
\end{tabular}

*Means provided for SRMH and SRH are presented on a scale of 1-5. In comparing means, a lower number is indicative of a better health rating. 
comorbidities, IADL impairments, and visits to personal doctor there is an increased proportion of those with better SRMH compared to SRH. For example, across comorbidity levels, $45 \%$ of participants with no medical conditions reported better SRMH, but over $70 \%$ of those with three or more conditions did. Looking at the mean values for SRMH and SRH, there appears to be a larger change towards worse SRH compared to SRMH with worsening health status. For example, across comorbidity levels, the difference in mean SRMH rating between zero and three comorbidities was 0.5 , while the difference in mean SRH rating between zero and three comorbidities was 1.1. In the table, similar but less dramatic trends can be seen with the other health or health service utilization variables (e.g., IADL impairments and visits to personal doctor).

\section{Discussion}

We sought to characterize the relationship between self-rated mental health and self-rated health in a large group of Medicare recipients. We found that, across a variety of sociodemographic and health-related characteristics, the same basic relationship held: it was most common for SRMH to be rated as better than SRH and uncommon for it to be rated as worse. These results are contrary to our hypothesis, based on previous research, that one variable would not show consistently better or worse ratings than the other. Instead, the relationship was skewed towards SRMH being better than SRH (as seen most clearly in Figure 1).

Even without drawing general conclusions about the meaning of the variables or their relationships, this observation encourages caution in the analytical use of SRMH in conjunction with SRH. SRH appears to have a floor effect on SRMH (i.e. SRMH is only rarely worse than SRH), which complicates assumptions about the effects of mental health as an independent construct. This raises the concern that collinearity could distort the effects of variables in analyses using both SRH and SRMH. Conclusions drawn about effects of $S R H$ or effects of SRMH in regression models that include both terms (i.e. that account for the other variable) would typically rely on the assumption that they are not highly collinear. Our analysis suggested that they are collinear, and in a skewed manner, which further complicates the interpretation. Thus, conclusions about the association of SRMH with other variables must thus be interpreted in light of their relationship with SRH.

Conclusions about the association of SRMH with other variables should also be interpreted with care given the absence of research about the psychometric properties of SRMH. Little is known about the validity and reliability of this measure, or how age may impact response over the lifespan. Some studies have suggested that SRH has significantly reduced predictive validity for mortality with increasing age, and in certain sociodemographic groups. ${ }^{19}$ It is possible that SRMH may have limited validity and/or reliability in certain populations as well.

Several factors might explain the skewed distribution of responses. First, it is possible that people in general consider that they have better mental health than physical health. Despite the simplicity of asking such a question, we do not know of any surveys that have inquired about the relationship in this way (i.e. Do you think your mental health is better than your physical health?). Some studies have suggested an intriguing paradox in aging whereby increased age is associated with better mental health despite failing physical health. ${ }^{20}$ As the majority of participants in the current study were aged 65 and older, the relationship between SRMH and SRH seen in the current study may be a reflection of this paradox. Suggested explanations for this paradox have included improved coping and emotional regulation, increased wisdom, and increased resilience. ${ }^{20}$ Second, those with mental disorders or symptoms may experience prejudice, discrimination, or stigma which can lead to downplaying of symptoms. ${ }^{21}$ It may be that participants were, in an effort to prevent stigma, unwilling to report poor mental health ratings as compared to overall health ratings. Third, mental health symptoms, such as depression, could introduce non-response bias. However, previous studies suggest depression would likely have a minimal effect on survey non-response. ${ }^{22,23}$ Fourth, mental health literacy may affect recognition of symptoms and impacts a participant's ability to identify the factors underlying mental health. ${ }^{24}$ Not only may symptom recognition be a challenge, but the SRMH question itself, which asks respondents to rate dimensions of emotional and/or mental health, may be a more difficult to respond to than SRH, because it asks participants to evaluate two dimensions of health rather than a single dimension. Fifth, the construct of the SRMH and SRH items might be responsible for differences in response. State-dependent reporting bias, also known as reporting heterogeneity, posits that different populations or sub-populations may interpret questions differently, and thus have systematically different thresholds between different ratings of health. ${ }^{25}$ Thus, a person's threshold for excellent SRMH may differ from another's and may also differ from their threshold for excellent SRH. Finally, placement order of questions and the presence of multiple health self-assessment questions within a single survey may impact how people assess their health. In a 2003 article examining data from CAHPS fee-for-service and managed care surveys, the Harvard Medicare Managed Care CAHPS Team suggested that placement of the SRMH variable immediately before the SRH variable influenced respondents by biasing ratings of their general health upward. ${ }^{26}$ While all of these explanations may be contributing to our observed findings, we do not expect the latter three alone to account for the observed relationship between SRMH and SRH. We conclude that, within a large group of Medicare recipients, the overwhelming trend was for recipients to consider themselves to be mentally at least just as healthy as compared to their overall health. This is a somewhat unexpected finding, and deserves further attention. It comports with other recent research indicating that mental health does not deteriorate, and may even improve, during aging. ${ }^{20}$

With some interesting exceptions, the subgroup analyses showed that, contrary to expectations, sociodemographic and medical factors seemed to have little association with the relative responses on SRMH and SRH items. One might reasonably conclude that the observed differences cannot be attributed to personlevel factors, and are instead general to the population. There were some unexpected differences between groups. More medical comorbidities, more IADL impairments, and more medical visits were associated with a greater likelihood of rating better SRMH than $\mathrm{SRH}$. This was particularly pronounced in comorbidities, where $45 \%$ of participants with no medical conditions reported better SRMH, but over $70 \%$ of those with three or more conditions did. Based on the mean values for SRMH and SRH, it appears that this effect occurs through an exaggerated worsening of SRH compared to SRMH with worsening health status. In other words, SRMH seems to be more resistant to the presence of comorbidities, IADL impairments, or medical visits than is SRH. This may point to a fundamental difference in how people formulate and differentiate between their mental and general health, with mental health being seen as removed from other health factors. This challenges the assumption, described in the introduction, that SRMH is a proxy for these general health factors.

To return to the analogy made in the introduction, the comparable relationship between dinner as a whole and dessert would be explained by the finding, Generally dessert is ranked better than the 
meal as a whole, and no matter how bad the meal was, the dessert was no worse. Other ways to phrase this might be At least dessert is usually pretty good or If dessert is bad, the whole meal was just as bad or worse. The condition that is not found is, The meal was great, except dessert was bad. Building on the same dinner analogy, it seems that dessert (SRMH) was typically rated as better than the whole meal in total (SRH), regardless of the context in which the meal was served. This raises the concern about the validity of treating $\mathrm{SRH}$ as an aggregate of subparts. It seems illogical that we would see this relationship if SRH is made up of subcomponents (physical, mental, functional, social, or other domains) which are averaged to produce a total score. We propose that SRH has been validated as a global measure of health, rather than being a composite measure of different parts that are averaged out, and that further study is needed in order to clarify the relationship between different ways of assessing health status.

Our findings introduce a general question about how older adults might define successful aging. In one study examining if older adults expected to age successfully, as defined by high cognitive and physical functioning, more participants expected worsening general health and functional dependence than expected depression. ${ }^{27}$ In other words, declines in mental health are not as frequently seen as a part of normal aging. Our results are consistent with this perspective, since with advancing age respondents seemed increasingly likely to describe differentially better mental than general health. One conceptual framework of successful aging that is quite consistent with our results involves three domains of health: physiological, psychological, and social. ${ }^{28}$ This model supports the idea that successful aging can coexist with medical comorbidity in the presence of emotional vitality, social engagement, and/or spirituality or religious commitment.

Our analyses have several limitations. First, the response rate was low, with over $55 \%$ of participants failing to respond to both $\mathrm{SRH}$ and SRMH questions. Beneficiary characteristics associated with nonresponse in the Medicare CAHPS survey include age younger than 45 years or older than 84 years, urban dwelling, nonWhite race/ethnicity, and dual eligibility for Medicaid. ${ }^{17}$ Second, the data is cross-sectional, and we cannot make any causal inferences about SRMH, SRH, and the other variables. Third, as $88 \%$ of participants were over aged over 65 years, conclusions drawn from our analyses should be limited to older age groups. Fourth, the data set does not include any measure of cognitive impairment. As age increases, so does the risk of cognitive impairment. Some data suggests that there may also be changes in meta-cognitive efficiency with increasing age. ${ }^{29}$ Given that the majority of respondents were over age 65 , cognitive changes could have impacted question comprehension and response. Finally, although we were able to look at the relationships between SRH and SRMH and physical comorbidities, we had no additional mental health measures to examine in the current study, such as symptoms of depression.

\section{Conclusions}

Most older adults in the CAHPS survey reported that their mental health was better than their general health. They rarely reported worse mental health than general health. This relationship did not seem associated with any particular sociodemographic or medical variables, and thus appears to be a general phenomenon. This finding encourages caution in the analytic use of self-rated mental health, and in particular adjustment for both it and self-rated general health. Additional research can help clarify the complex relationship between these variables.
Correspondence: Elena Malaika Magwene, Puget Sound VA Medical Center, GRECC S-182, Seattle, WA 98108, USA.

Tel.: + 1.206.422.9005.

E-mail: emagwene@u.washington.edu

Key words: Self-rated mental health, self-rated health, CAHPS, Older Adults. Contributions: EMM, ST, conception or design of the work, data collection, drafting the article; EMM, ARQ, GLM, LKM, ST, data analysis and interpretation, critical revision of the article, final approval of the version to be published.

Conflict of interest: the authors declare no potential conflict of interest. Acknowledgments: the research conducted for this manuscript was supported by the National Institutes of Health. Dr. Marshall is funded by a K01 career development award (Grant \#5K01AG048416-03) from the National Institutes of Aging.

Received for publication: 5 May 2017.

Accepted for publication: 11 August 2017.

(C) Copyright E.M. Magwene et al., 2017

Licensee PAGEPress, Italy

Journal of Public Health Research 2017;6:967

doi:10.4081/jphr.2017.967

This work is licensed under a Creative Commons Attribution NonCommercial 4.0 License (CC BY-NC 4.0).

\section{References}

1. DeSalvo KB, Fan VS, McDonell MB, Fihn SD. Predicting mortality and healthcare utilization with a single question. Health Serv Res 2005;40:1234-46.

2. Idler EL, Kasl SV. Self-ratings of health: do they also predict change in functional ability? J Gerontol B Psychol Sci Soc Sci 1995;50:S344-53.

3. Nabalamba A, Millar WJ. Going to the doctor. Health Rep 2007;18:23-35.

4. Halpern MT, Urato MP, Kent EE. The health care experience of patients with cancer during the last year of life: analysis of the SEER-CAHPS data set. Cancer 2017;123:336-44.

5. Kim G, DeCoster J, Chiriboga DA, et al. Associations between self-rated mental health and psychiatric disorders among older adults: do racial/ethnic differences exist? Am J Geriatr Psychiatry 2011;19:416-22.

6. Mawani FN, Gilmour H. Validation of self-rated mental health. Health Rep 2010;21:61-75.

7. Dogra S, Baker J. Physical activity and health in Canadian asthmatics. J Asthma 2006;43:795-9.

8. Park J, Knudson S. Medically unexplained physical symptoms. Health Rep 2007;18:43-7.

9. Zuvekas SH, Fleishman JA. Self-rated mental health and racial/ethnic disparities in mental health service use. Med Care 2008;46:915-23.

10. Vasiliadis HM, Tempier R, Lesage A, Kates N. General practice and mental health care: determinants of outpatient service use. Can J Psychiatry 2009;54:468-76.

11. Ahmad F, Jhajj AK, Stewart DE, et al. Single item measures of self-rated mental health: a scoping review. BMC Health Serv Res 2014;14:398.

12. Fleishman JA, Zuvekas SH. Global self-rated mental health: associations with other mental health measures and with role functioning. Med Care 2007;45:602-9.

13. Levinson D, Kaplan G. What does self rated mental health represent. J Public Health Res 2014;3:287.

14. Dembosky JW, Haviland AM, Elliott M, et al. Does naming the 
focal plan in a CAHPS survey of health care quality affect response rates and beneficiary evaluations? Publ Opin Q 2013;77:455-73.

15. Hatfield LA, Zaslavsky AM. Implications of variation in the relationships between beneficiary characteristics and medicare advantage CAHPS measures. Health Serv Res 2017;52:131029.

16. Hays RD, Martino S, Brown JA, et al. Evaluation of a Care Coordination Measure for the Consumer Assessment of Healthcare Providers and Systems (CAHPS) Medicare survey. Med Care Res Rev 2014;71:192-202.

17. Klein DJ, Elliott MN, Haviland AM, et al. Understanding nonresponse to the 2007 Medicare CAHPS survey. Gerontologist 2011;51:843-55.

18. Goldstein E, Cleary PD, Langwell KM, et al. Medicare managed care CAHPS(R): a tool for performance improvement. Health Care Financ Rev 2001;22:101-7.

19. Zajacova A, Woo H. Examination of age variations in the predictive validity of self-rated health. J Gerontol B Psychol Sci Soc Sci 2016;71:551-7.

20. Thomas ML, Kaufmann CN, Palmer BW, et al. Paradoxical trend for improvement in mental health with aging: a community-based study of 1,546 adults aged 21-100 years. J Clin Psychiatry 2016;77:e1019-25.

21. Oexle N, Ajdacic-Gross V, Kilian R, et al. Mental illness stigma, secrecy and suicidal ideation. Epidemiol Psychiatr Sci
2015:1-8.

22. Etter JF, Perneger TV. Analysis of non-response bias in a mailed health survey. J Clin Epidemiol 1997;50:1123-8.

23. Volken T. Second-stage non-response in the Swiss health survey: determinants and bias in outcomes. BMC Public Health 2013; 13:167.

24. Goldney RD, Fisher LJ, Wilson DH. Mental health literacy: an impediment to the optimum treatment of major depression in the community. J Affect Disord 2001;64:277-84.

25. Lindeboom M, van Doorslaer E. Cut-point shift and index shift in self-reported health. J Health Econ 2004;23:1083-99.

26. Changes in the general health status (GHS) and general mental health $(\mathrm{GMH})$ variables in the CAHPS Medicare satisfaction surveys: Centers for Medicare and Medicaid Services; 2003 [Available from: https:/www.cms.gov/Research-StatisticsData-and-Systems/Research/CAHPS/ Downloads/03_Chgs GHSandGMHVarRPT.pdf.

27. Sarkisian CA, Hays RD, Berry SH, Mangione CM. Expectations regarding aging among older adults and physicians who care for older adults. Med Care 2001;39:1025-36.

28. Young Y, Frick KD, Phelan EA. Can successful aging and chronic illness coexist in the same individual? A multidimensional concept of successful aging. J Am Med Dir Assoc 2009; 10:87-92.

29. Palmer EC, David AS, Fleming SM. Effects of age on metacognitive efficiency. Conscious Cogn 2014;28:151-60. 